\title{
Interface Trap Density Estimation in FinFETs from the Subthreshold Current
}

\author{
J. Schmitz, Senior Member, IEEE, B. Kaleli, P. Kuipers, N. van den Berg, S.M. Smits, \\ and R.J.E. Hueting, Senior Member, IEEE
}

\begin{abstract}
In this work we present a measurement approach to determine the interface trap density in FinFETs as a function of their energy. It is based on the precise determination of the gate voltage dependent ideality factor of the subthreshold current in this device. The required measurement accuracy for temperature, drain current and transconductance is derived, and we propose an implementation for wafer-level device measurement on contemporary test set-ups. Exemplary interface trap distributions are shown as obtained from two FinFET device technologies, featuring the commonly observed bathtub shape.
\end{abstract}

Index Terms-MOS devices, FinFETs, Current, Complementary MOSFETs (CMOSFETs), Traps, Interface states

\section{INTRODUCTION}

At the semiconductor-insulator interface in a metal-oxidesemiconductor (MOS) stack, imperfections in the chemical structure lead to interface traps. These localized defects can trap and release mobile charge, leading to drain-current noise [1][2] as well as a reduced electrostatic control of the gate over the channel [3]-[5]. Interface traps are formed during device processing, but can be additionally created by e.g. ionizing radiation and electrical stress. CMOS process development, process control and reliability assessment require an accurate estimate of the interface state density in these devices.

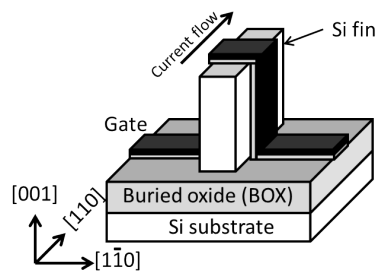

(a)

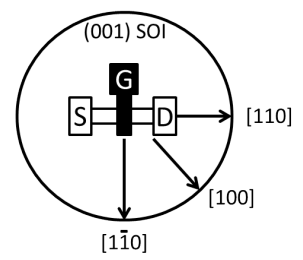

(b)
Fig. 1. (a) Bird's eye view of a schematic single fin SOI (110)/[110] FinFET and (b) its orientation on the silicon wafer. The fin has source and drain connections, and the channel region is surrounded by the gate dielectric and the gate metal. In this device configuration there is no separate body contact. Two device sets were used for the experiments: device set I in which the fin height $H_{\text {FIN }}$ is $65 \mathrm{~nm}$, and device set II where $H_{\text {FIN }}=150 \mathrm{~nm}$. The figures are not to scale.

The authors are with the MESA+ Institute for Nanotechnology, University of Twente, Enschede, The Netherlands. B. Kaleli is now with ASML, Veldhoven, The Netherlands. N. van den Berg is now with Micronit Microfluidics, Enschede, The Netherlands. Corresponding author: Jurriaan Schmitz, University of Twente, 7500 AE Enschede, The Netherlands (email: j.schmitz@utwente.nl). Part of this work was financially supported by the Dutch Technology Foundation STW, an applied science division of NWO and the Technology Program of the Ministry of Economic Affairs.
Several methods are known to experimentally estimate the interface trap density of states $\left(D_{\mathrm{it}}\right)$ [3],[4], such as the lowfrequency (quasi-static) capacitance-voltage $(C-V)$ method, the conductance method, the high-frequency $C-V$ method, and charge pumping. Some of these require specific test equipment, and they all require a separate backgate or body contact. The latter, in particular, is a problem in many device configurations such as FinFETs because separate body contacts cannot be realized, see for instance Fig. 1.

Therefore, we propose to extract the interface trap density from subthreshold current measurements, extending a technique earlier proposed for bulk MOSFETs [6]. In the remainder of this article we will refer to the new method as the $g_{\mathrm{m}} / I_{\mathrm{D}}$ method.

Prior art related to this work deals with subthreshold current modeling of planar SOI MOSFETs [7]-[11] in which there is an unavoidable asymmetry between the trap densities at the gate dielectric-SOI and SOI-buried-oxide (BOX) interfaces. In some of these reports analytical models have been used for extracting the $D_{\text {it }}$ in FD-SOI MOSFETs [10], [11]. Also in the field of GAA Si nanowire FETs analytical models have been used with [12],[13] or without [14] the aid of numerical simulation tools.

In this work, we focus on the measurement issues in the $g_{\mathrm{m}} / I_{\mathrm{D}}$ method for $D_{\text {it }}$ estimation of fully-depleted symmetric FETs. In section II the basic methodology is briefly introduced. Section III covers the experimental details and the results. Section IV describes the accuracy of the method and the necessary measurement precision, after which the conclusions are drawn in section $\mathrm{V}$.

\section{RELATION BETWEEN SUBTHRESHOLD CURRENT AND TRAP DENSITY}

In this section we postulate a newly derived relationship between the subthreshold current and the interface state density. It is based on the assumptions that the depletion charge can be neglected in FinFETs, and that the transistor under study shows negligible short-channel effects. Further, the trap distribution is assumed to be uniform along the channel. For subthreshold, we derived that the ideality factor $m$ follows:

$m=\frac{d V_{\mathrm{GS}}}{d \psi_{\mathrm{s}}}=\frac{1}{u_{T}} \frac{I_{\mathrm{D}}}{g_{\mathrm{m}}}=1+\frac{d}{d \psi_{\mathrm{s}}} \int_{0}^{\psi_{\mathrm{s}}} \frac{C_{\mathrm{it}}(\psi)}{C_{\mathrm{ins}}} d \psi=\frac{C_{\mathrm{ins}}+C_{\mathrm{it}}\left(\psi_{\mathrm{s}}\right)}{C_{\mathrm{ins}}}$

Hence,

$$
C_{\mathrm{it}}\left(\psi_{\mathrm{s}}\right)=\left(\frac{1}{u_{T}} \frac{I_{\mathrm{D}}\left(\psi_{\mathrm{s}}\right)}{g_{\mathrm{m}}\left(\psi_{\mathrm{s}}\right)}-1\right) C_{\mathrm{ins}}
$$


In these equations, $V_{\mathrm{GS}}$ is the gate-source voltage, $\psi_{\mathrm{s}}$ is the surface potential, $u_{T}=k T / q$ is the thermal voltage, $I_{\mathrm{D}}$ the drain current, $g_{\mathrm{m}}$ the transconductance, $C_{\mathrm{it}}$ the interface trap capacitance per unit area, and $C_{\mathrm{ins}}$ is the insulator capacitance per unit area. Because equation 2 is used to derive $C_{\mathrm{it}}$ from the ratio between transconductance and drain current, we name this approach the $g_{\mathrm{m}} / I_{\mathrm{D}}$ method. The trap density follows from [3]:

$$
D_{\mathrm{it}}=\frac{1}{q^{2}} C_{\mathrm{it}} .
$$

Further, since at the mid gap energy level $\psi_{\mathrm{s}}=0 \mathrm{~V}$ we can assume that

$$
E-E_{\mathrm{v}} \approx \frac{E_{\mathrm{g}}}{2}+q \cdot \psi_{\mathrm{s}},
$$

ignoring the contribution of the effective densities of band states and where $E, E_{\mathrm{v}}$, and $E_{\mathrm{g}}$ are the energy, valence band edge energy, and bandgap, respectively.

Equation (4) is valid provided that the (electron) quasiFermi level does not vary much along the channel length so that the same energy level $E$ is probed across the entire channel. This implies that the method works best for a low applied $V_{\text {DS }}$. On the other hand, a certain drain-source bias is of course necessary to measure the subthreshold current. At a given measurement temperature $T$ it is recommended not to exceed $V_{\mathrm{DS}} \simeq u_{T}$.

We further need to relate the surface potential $\psi_{\mathrm{s}}$ to the applied gate voltage $V_{\mathrm{GS}}$. To that purpose, we employ the longchannel subthreshold current relation [3], [5], [15]:

$$
I_{\mathrm{D}}=I_{0} \cdot e^{\frac{\psi_{\mathrm{S}}}{u_{T}}}\left(1-e^{-\frac{V_{\mathrm{DS}}}{u_{T}}}\right),
$$

where $V_{\mathrm{DS}}$ is the drain-source voltage, and

$$
I_{0}=q \mu_{\mathrm{n}} n_{\mathrm{i}} u_{T} \cdot \frac{N_{\text {FIN }} A_{\text {body }}}{L}
$$

with $A_{\text {body }}=W_{\text {FIN }} \cdot H_{\text {FIN }}$ and $A_{\text {body }}=\pi \cdot R^{2}$ for Fin- and nanowire-FETs [16], [17], respectively. $\mu_{\mathrm{n}}, N_{\mathrm{FIN}}, W_{\mathrm{FIN}}, H_{\mathrm{FIN}}$, $R$ and $L$ are the electron mobility, amount of wires or fins, fin width, fin height, nanowire radius and channel length, respectively.

We derive $\psi_{\mathrm{s}}$ from $I_{\mathrm{D}}$ using equations (5) and (6) for each applied bias. Then, using equations (1)-(4) it is possible to construct the trap density as a function of $E-E_{\mathrm{v}}$ from the subthreshold $I-V$ curve. The derivation of the equations above, and a rigorous test of the approach using TCAD simulations, are the topic of a paper in print [18]. In this article we discuss the characterization aspects of the $g_{\mathrm{m}} / I_{\mathrm{D}}$ technique with a particular focus on the accuracy of the obtained results.

\section{EXPERIMENTAL}

We characterized FinFETs from two fabrication lines to investigate the $g_{\mathrm{m}} / I_{\mathrm{D}}$ method experimentally. The first set of SOI FinFETs, device set I, features an $\mathrm{HfSiO}$ gate dielectric with an effective oxide thickness of $1.6 \mathrm{~nm}$ and a TiN gate capped with polysilicon. The fin height is $65 \mathrm{~nm}$. For the processing details, see [19].

The second set of SOI FinFETs, device set II, features $150 \mathrm{~nm}$ high $\mathrm{Si}$ fins, an $11 \mathrm{~nm}$ thermally grown $\mathrm{SiO}_{2}$ gate oxide and a TiN gate capped with polysilicon. The process details are documented in [20].

The devices studied in this paper have the $(1 \overline{1} 0) /[110]$

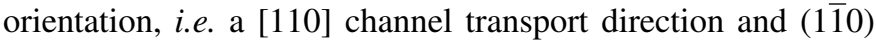
oriented sidewalls, as depicted in Fig. 1; and comprise 5 parallel fins $\left(N_{\text {FIN }}=5\right)$.

Fig. 2 shows exemplary current-voltage $\left(I_{\mathrm{D}}-V_{\mathrm{GS}}\right)$ characteristics for various fin and gate dimensions for both sets of devices. The characteristics were recorded with a Keithley 4200 semiconductor parameter analyzer and a Cascade probe station at $V_{\mathrm{DS}}=25 \mathrm{mV}$. For all measurements the temperature $T$ was fixed at $300 \mathrm{~K}$ using a low temperature chuck (PM300) of Advanced Temperature Test Systems (ATT Systems). All studied devices had negligible short-channel effects.
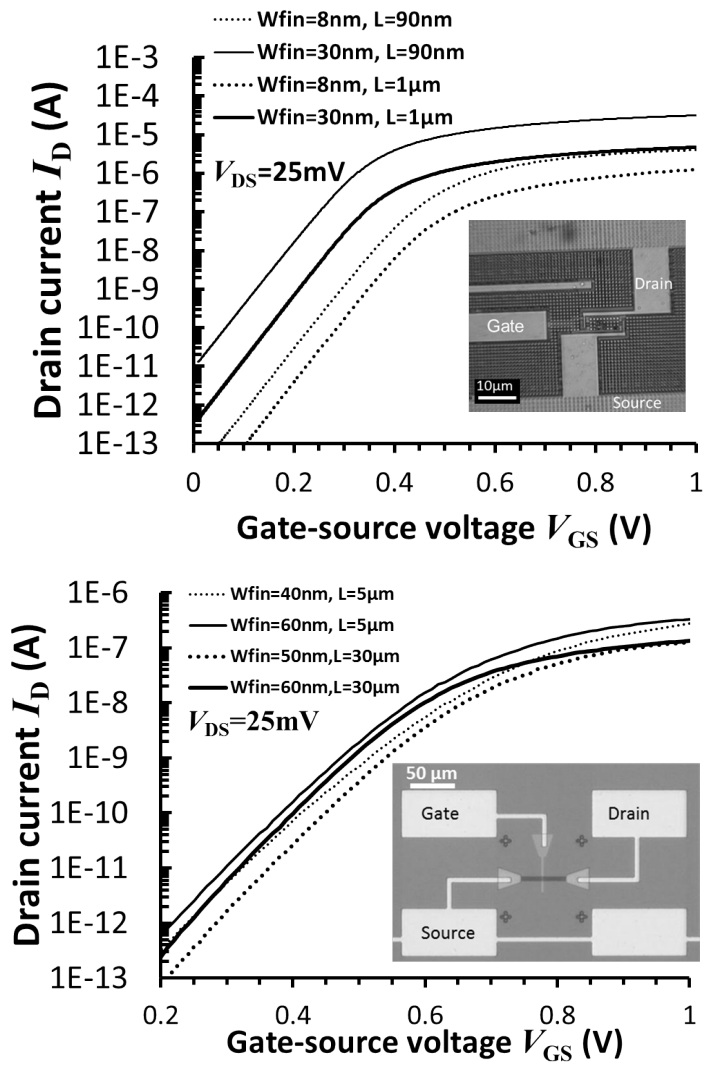

Fig. 2. $I_{\mathrm{D}}-V_{\mathrm{GS}}$ characteristics of the fabricated FinFETs $\left(V_{\mathrm{DS}}=25 \mathrm{mV}\right)$ for various fin widths ( $\left.W_{\text {FIN }}\right)$ and gate lengths for (a) device set I and (b) device set II. The insets are optical micrographs of a 5-fin FinFET.

For analyzing the electrical results we used pre-amplifiers which enabled us to measure currents down to the $10 \mathrm{fA}$ range. The step size in $V_{\mathrm{GS}}$ was fixed to $0.5 \mathrm{mV}\left(\Delta V_{\mathrm{GS}}=0.5 \mathrm{mV}\right)$. We used a running least squares fit on 41 consecutive measurements, fitting a linear function on $\ln \left(I_{\mathrm{D}}\right)$ versus $V_{\mathrm{GS}}$, to obtain precise values of $g_{\mathrm{m}}$ and $I_{\mathrm{D}}$ by averaging out measurement noise. From these values the surface potential itself, and the surface potential dependent ideality factor $m$ were computed. Some of these results are shown in Fig. 3.

The results indicate that for device set I the ideality factor $m \approx 1$, and starts to increase from $V_{\mathrm{GS}}=0.2 \mathrm{~V}$ onwards. For the device set II $m$ is higher, typically starting from around 1.5 and monotonously increasing. The more noisy curves could be 
related to the relatively high trap density. Spikes, as visible in Fig. 3b, occur randomly on some devices and are likely a measurement artifact. The curves in Fig. 3 cover mainly the subthreshold regime.
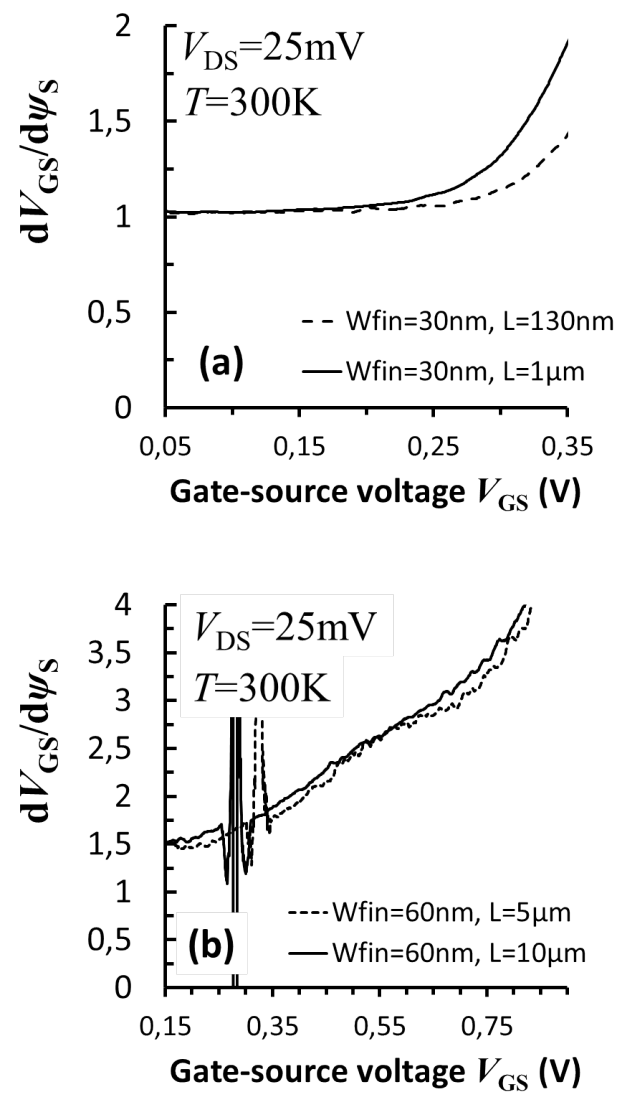

Fig. 3. Extracted ideality factors against $V_{\mathrm{GS}}$ for (a) $W_{\mathrm{FIN}}=30 \mathrm{~nm}$ (device set I), and (b) $W_{\text {FIN }}=60 \mathrm{~nm}$ (device set II).

For estimating $D_{\text {it }}(E)$ equations (2)-(4) have been used as shown in Fig 4. As can be seen traditional bathtub-shape curves have been obtained ranging from around $0.15 \mathrm{eV}$ above mid gap up to the conduction band edge; for the lower energy levels the results appear rather noisy. By plotting the same curves on semilog scale (Fig. $4 \mathrm{~b}$ ) the minimum $D_{\text {it }}$ values can be determined which are around $3 \cdot 10^{11} \mathrm{~cm}^{-2} \mathrm{eV}^{-1}$ for $W_{\text {FIN }}=20$ and $30 \mathrm{~nm}$. For lower energy levels between 0.7 and $0.85 \mathrm{eV}$ the trap density for $W_{\text {FIN }}=8 \mathrm{~nm}$ could not be measured, indicating lower values.

Fig. 5 shows some results obtained on device set II for a gate length of $5 \mu \mathrm{m}$. The results indicate higher $D_{\text {it }}$ values than for device set I, irrespective of the gate length (not shown) or fin width. For device set II the technique loses resolution towards midgap due to measurement accuracy, while for device set I it is due to gate-induced drain leakage.

\section{ACCURACY OF THE METHOD}

Measurement errors propagate into the $D_{\text {it }}(E)$ determination, and the presented technique is quite sensitive to such errors. In this section we discuss the main causes of erroneous $D_{\text {it }}$ determination and their mitigation.
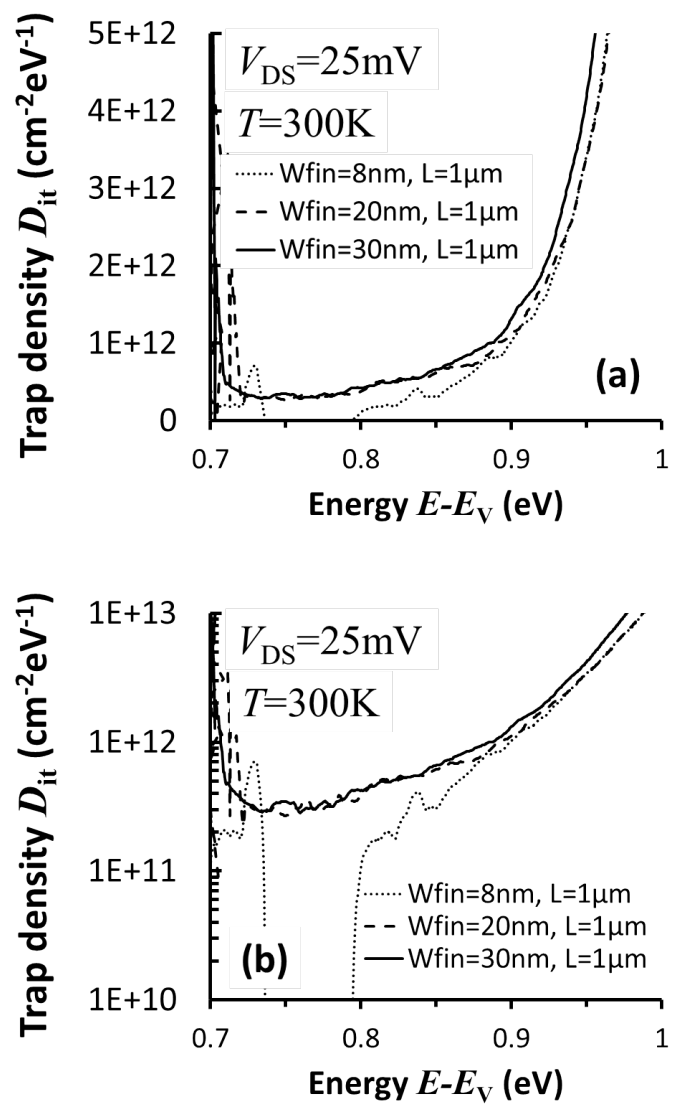

Fig. 4. Extracted trap density $D_{\text {it }}$ against the energy $E-E_{\mathrm{v}}$ for device set I using equations (2) and (4). (a) linear scale, and (b) semi-log scale.

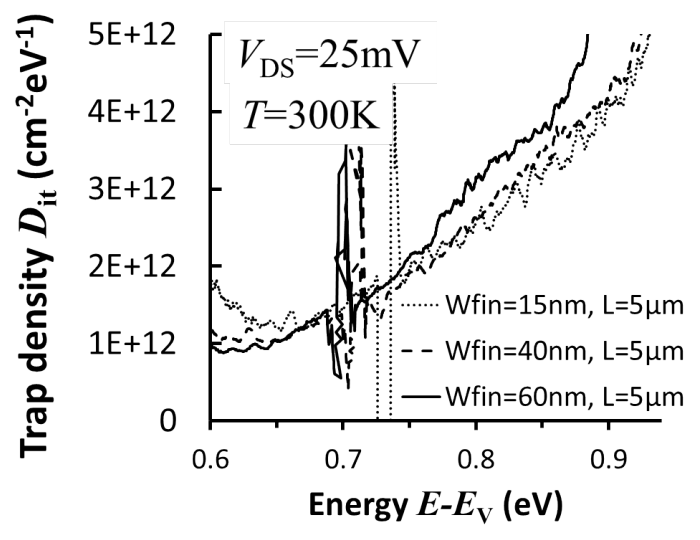

Fig. 5. Extracted trap density $D_{\text {it }}$ against the energy $E-E_{\mathrm{V}}$ for device set II using equations (2) and (4). The gate length is $5 \mu \mathrm{m}$.

Three parameters require precise determination for the $g_{\mathrm{m}} / I_{\mathrm{D}}$ method to be accurate: the device temperature $T$, drain current $I_{\mathrm{D}}$, and transconductance $g_{\mathrm{m}}$. The $D_{\text {it }}$ itself is sensitive to all three; the trap energy $\left(E-E_{\mathrm{v}}\right)$ relies on the accurate determination of $T$ and $I_{\mathrm{D}}$, but is much less sensitive to measurement errors. We therefore focus this discussion on the effect of errors of these three parameters on $D_{\mathrm{it}}$ as determined using equations (2) and (3). 
From equation (2) and using $g_{\mathrm{m}}=I_{\mathrm{D}} / m u_{T}$ we can derive that an error in $I_{\mathrm{D}}$ measurement, $\delta I_{\mathrm{D}}$, leads to an error $\delta D_{\text {it }}$ following:

$$
\delta D_{\mathrm{it}}=\frac{m C_{\mathrm{ins}}}{q^{2}} \frac{\delta I_{\mathrm{D}}}{I_{\mathrm{D}}} \approx \frac{m C_{\mathrm{ins}}}{q^{2}} \frac{\delta g_{\mathrm{m}}}{g_{\mathrm{m}}} .
$$

Because $I_{\mathrm{D}}$ and $g_{\mathrm{m}}$ are extracted from the same data points, it is not safe to assume that $\delta I_{\mathrm{D}}$ and $\delta g_{\mathrm{m}}$ are uncorrelated. Assuming $m=1$ and an EOT of $1 \mathrm{~nm}$, a 100-ppm error on the drain current measurement will lead to an error on $D_{\text {it }}$ of $2.2 \times 10^{9} \mathrm{~cm}^{-2} \mathrm{eV}^{-1}$. The same can be stated for the $g_{\mathrm{m}}$ determination. Relative measurement errors in the 100-ppm regime are readily obtained with wafer-level current measurements, also with automatic prober systems (see e.g. [21]). However care must be taken in the low current regime below $1 \mathrm{nA}$ where the accuracy tends to deteriorate. For an accurate determination of interface trap density nearer to midgap, test structures with many parallel fins are therefore recommended, to obtain higher overall subthreshold currents. However such test structures were not available for this work.

Similarly, we find the impact of a temperature error $\delta T$ to be approximated by:

$$
\delta D_{\mathrm{it}} \approx \frac{m C_{\mathrm{ins}}}{q^{2}} \frac{\delta T}{T} .
$$

This is a critical factor in the $D_{\text {it }}$ extraction. An error of the device temperature as specified for our PM300 thermochuck, $0.6 \mathrm{~K}$, with the same assumptions as above, leads to an error in the $D_{\text {it }}$ determination of $4.4 \times 10^{10} \mathrm{~cm}^{-2} \mathrm{eV}^{-1}$. Clearly, a more accurate temperature-controlled chuck in equilibrium is advised - modern thermo-chuck systems control the wafer temperature better than $0.1 \mathrm{~K}$. Alternatively, the local temperature can be recorded during measurement by different means. Self-heating of the transistor can likely be ignored in the measurement as we work in subthreshold.

As the accuracy of this $D_{\text {it }}$ measurement technique then reaches $10^{10} \mathrm{~cm}^{-2} \mathrm{eV}^{-1}$ or better, it becomes possible to study single traps in small finFETs (as long as they exhibit longchannel characteristics). We speculate that the $g_{\mathrm{m}} / I_{\mathrm{D}}$ technique proposed here can thus be used to study single-trap behavior in more detail, as well as possible $D_{\text {it }}$ variability issues in this type of devices.

Besides experimental accuracy, it is reported in literature that the subthreshold current method becomes inaccurate in case of doping nonuniformity [3], [4] and non-uniform oxide charge [6]. For fully-depleted devices with low body doping the first issue is circumvented. Non-uniform oxide charge may still be a factor of concern.

\section{CONCLUSIONS}

This article presents the characterization aspects of the newly developed $g_{\mathrm{m}} / I_{\mathrm{D}}$ method to extract $D_{\text {it }}$ of symmetric fully depleted field-effect transistors from the subthreshold current. Our experimental data show a traditional bathtubshape like trap density against the energy ranging from around $0.15 \mathrm{eV}$ above mid-gap up to the conduction band edge. An error propagation analysis shows that particular care is required in the device temperature measurement and $g_{\mathrm{m}}$ determination to obtain accurate results. An accuracy better than $10^{10} \mathrm{~cm}^{-2} \mathrm{eV}^{-1}$ can be obtained with contemporary waferlevel characterization tools.

\section{REFERENCES}

[1] K.K. Hung, P. K. Ko, C. Hu and Y. C. Cheng, "A unified model for the flicker noise in metal-oxide-semiconductor field effect transistors", IEEE Trans. Electron Devices, vol. 37 no. 3, pp. 654-665 (1990).

[2] G. Ghibaudo, O. Roux, Ch. Nguyen-Duc, F. Balestra, and J. Brini, "Improved analysis of low frequency noise in field-effect MOS transistors", Physica status solidi A, vol. 124, pp. 571-581, 1991.

[3] D.K. Schroder, "Semiconductor material and device characterization", $3^{\text {rd }}$ edition, John Wiley \& Sons, Inc., USA, 2006.

[4] S.C. Witczak, J.S. Suehle, and M. Gaitan, "An experimental comparison of measurement techniques to extract $\mathrm{Si}_{-} \mathrm{SiO}_{2}$ interface trap density", Solid-State Electronics, vol. 35, no. 3, pp. 345-355, 1992.

[5] S.M. Sze and K.K. Ng, "Physics of Semiconductor Devices", $3^{\text {rd }}$ edition, John Wiley \& Sons, Inc., USA, 2007.

[6] R.J. van Overstraeten, G.J. DeCleck, and P.A. Muls, "Theory of the MOS transistor in weak inversion - new method to determine the number of surface states", IEEE Trans. Electron Devices, vol. ED-22, no. 5, pp. 282-288, 1975.

[7] J.-P. Colinge, "Subthreshold slope of thin-film SOI MOSFET's", IEEE Electron Device Lett., vol. EDL-7, no. 4, pp. 244-246, 1986.

[8] D.J. Wouters, J.-P. Colinge, and H.E. Maes, "Subthreshold slope in thin-film SOI MOSFET's", IEEE Trans. Electron Devices, vol. 37, no. 9, pp. 2022-2033, 1990.

[9] F. Balestra, M. Benachir, J. Brini, and G. Ghibaudo, "Analytical models of subthreshold swing and threshold voltage for thin- and ultra-thin-film SOI MOSFETs", IEEE Trans. Electron Devices, vol. 37, no. 11, pp. 2303-2311, 1990.

[10] Z. Lun, D.S. Ang, and C.H. Ling, "A novel subthreshold slope technique for the extraction of the buried-oxide interface trap density in the fully depleted SOI MOSFET", IEEE Electron Device Lett., vol. 21, no. 8, pp. 411-413, 2000.

[11] J.-Y. Cheng, C.W. Yeung, and C. Hu, "Extraction of front and buried oxide interface trap densities in fully depleted silicon-on-insulator metal-oxide-semiconductor field-effect transistor", ECS Solid State Lett., vol. 2, no. 5, pp. Q32-Q34, 2013.

[12] S. Sato et al., "Extraction of additional interfacial states of silicon nanowire field-effect transistors", Appl. Phys. Lett., vol. 98, pp. 233506-1-3, 2011

[13] F. Najam et al., "Interface trap density of gate-all-around silicon nanowire field-effect transistors with TiN gate: extraction and compact model", IEEE Trans. Electron Devices, vol. 60, no. 8, pp. 2457-2463, 2013.

[14] B.H. Hong et al., "Subthreshold degradation of gate-all-around silicon nanowire field-effect transistors: effect of interface trap charge", IEEE Electron Device Lett., vol. 32, no. 9, pp. 1179-1181, 2011.

[15] P.A. Muls, G.J. DeClerck, and R.J. van Overstraeten, "Characterization of the MOSFET operating in weak inversion", $A d v$. in Electron. and Electron Phys., vol. 47, pp. 197-266, 1978.

[16] Y. Taur, X. Liang, W. Wang, and H. Lu, "A continuous, analytic drain-current model for DG MOSFETs", IEEE Electron Device Lett., vol. 25, no. 2, pp. 107-109, 2004.

[17] D. Jiménez et al., "A continuous, analytic $I-V$ model for surrounding-gate MOSFETs", IEEE Electron Device Lett., vol. 25, no. 8, pp. 571-573, 2004.

[18] B.K. Boksteen, J. Schmitz and R.J.E. Hueting, "Interface trap density estimation in FinFETs using the $g_{\mathrm{m}} / I_{\mathrm{D}}$ method in the subthreshold regime", IEEE Trans. Electron Devices, in print (2016).

[19] M.J.H. van Dal et al., "Highly manufacturable FinFETs with sub-10nm fin width and high aspect ratio fabricated with immersion lithography”, IEEE VLSI Technology Symp., pp. 110-111, 2007.

[20] B. Kaleli, "Characterization of strained silicon FinFETs and the integration of a piezoelectric layer", PhD thesis, University of Twente, Enschede, The Netherlands, 2013.

[21] J. Schmitz and H. P. Tuinhout, "A study of measurement system noise for soft breakdown triggering", Proc. IEEE ICMTS, pp. 99-102, 2001. 